\title{
miR-33a is downregulated in melanoma cells and modulates cell proliferation by targeting PCTAIRE1
}

\author{
FANGZHEN TIAN $^{1 *}$, HONGTU WEI $^{2 *}$, HUA TIAN $^{3}$, YING QIU $^{1}$ and JIAN XU ${ }^{4}$ \\ Departments of ${ }^{1}$ Dermatology and ${ }^{2}$ Orthopedics, Jining No. 1 People's Hospital, Jining, Shandong 272000; \\ ${ }^{3}$ Department of Public Health, Jining Beihu Hospital, Jining, Shandong 272067; \\ ${ }^{4}$ Department of Public Health, Center for Disease Control and Prevention of Jining City, Jining, Shandong 272113, P.R. China
}

Received February 19, 2015; Accepted January 26, 2016

DOI: $10.3892 / \mathrm{ol} .2016 .4321$

\begin{abstract}
MicroRNA-33a (miR-33a) was previously identified as a lipid regulator that controls the cellular balance between cholesterol and fatty acid metabolism. However, its role in tumor progression is largely unknown. The present study identified that miR-33a acts as a tumor suppressor in melanoma cells. The present study revealed that miR-33a was downregulated in melanoma cells compared with melanocytes. Overexpression of miR-33a suppressed the colony formation of human melanoma SK-MEL-1 and WM-115 cells. Furthermore, a bromodeoxyuridine incorporation assay and anaphase analysis revealed that miR-33a inhibits melanoma cell proliferation. miR-33a overexpression inhibited p27 phosphorylation and upregulated p27 expression. Additionally, the present study demonstrated that PCTAIRE1 was a direct target of miR-33a; miR-33a overexpression suppressed the luciferase activity of a reporter construct containing a 3'-untranslated region of PCTAIRE1 and downregulated PCTAIRE1 in melanoma cells. An overexpression of PCTAIRE1 reversed the miR-33a-induced p27 accumulation and tumor suppressive effects. In summary, the present findings offer novel mechanistic insights into miR-33a and its downstream target in melanoma cells.
\end{abstract}

\section{Introduction}

Skin cancer is the most common malignancy diagnosed in the United States, with 3.5 million melanomas diagnosed in 2 million individuals annually (1). Melanoma is a skin cancer characterized by the abnormal proliferation of melanocytes that invade the basement membrane (2). Although melanoma

Correspondence to: Dr Hongtu Wei, Department of Orthopedics, Jining No. 1 People's Hospital, 6 Jiankang Road, Jining, Shandong 272000, P.R. China

E-mail: hongtongweijn@126.com

*Contributed equally

Key words: miR-33a, PCTAIRE1, melanoma, p27 accounts for $<5 \%$ of skin cancers, it results in the highest number of mortalities (1-3). The lack of a therapeutic response in patients that receive the currently available treatments highlights the importance of an improved understanding of the complex molecular mechanisms that contribute to melanoma development.

MicroRNAs (miRNAs) are a family of noncoding RNAs $\sim 22$ nucleotides in length, which may suppress gene expression by pairing to the 3 '-untranslated region (3'-UTR) of target messenger (m)RNAs (4-6). The extent of base pairing between miRNA and mRNA appears to determine the balance between the cleavage and degradation of mRNA (7). Currently, it is widely accepted that miRNA alterations are involved in the initiation and progression of human cancer (4). miRNA-33 (miR-33) is a newly characterized miRNA located within the intronic sequences of the sterol regulatory element-binding protein (SREBP) genes, which regulate cholesterol and fatty acid metabolism in combination with their host genes (8-11). There are two miR-33 genes in humans, consisting of miR-33a and miR-33b. miR-33a and miR-33b are localized in corresponding introns of the SREBP2 and SREBP1 genes, respectively. Previous studies have focused on the metabolic function of miR-33. The role of miR-33 in tumor biology has been identified (12-15), yet its function in melanoma remains unclear.

PCTAIRE1, also termed cyclin-dependent kinase 16 (CDK16), is a serine/threonine kinase that was originally identified as a cyclin dependent kinase 1-like kinase $(16,17)$. It has been reported that PCTAIRE1 is involved in vesicular exocytosis, protein secretion, neuronal migration, neurite outgrowth and spermatogenesis (18-21). A recent study revealed that PCTAIRE1 phosphorylates p27 and promotes tumorigenesis in liver and breast cancer (22). The present study investigated the potential role of miR-33a in melanoma, and identified that the miRNA acts as a tumor suppressor.

\section{Materials and methods}

Cell lines. Human melanoma SK-MEL-1 and WM-115 cell lines were purchased from the American Type Culture Collection (ATCC; Manassas, VA, USA) and maintained in Eagle's minimum essential medium (Gibco; Thermo Fisher Scientific, Inc., Waltham, MA, USA) supplemented with $10 \%$ fetal bovine serum (Gibco; Thermo Fisher Scientific, Inc.). Primary 
epidermal melanocytes (ATCC; PEMI, catalog number PCS-200-012 ${ }^{\mathrm{TM}}$; PEM2, PCS-200-013 ${ }^{\mathrm{TM}}$ ) were maintained following ATCC protocol.

Lentivirus infection. Pre-miR-33a expression and negative control lentivirus was obtained from GeneChem Co., Ltd. (Shanghai, China). SK-MEL-1 and WM-115 cells were plated on a 6-well plate (Corning Incorporated, Corning, NY, USA) and lentivirus was added in medium with $5 \mu \mathrm{g} / \mathrm{ml}$ polybrene (Solarbio Science \& Technology Co., Ltd., Beijing, China). After $12 \mathrm{~h}$, the media was replaced with fresh medium. Stable infection cell strains were selected by puromycin (Solarbio Science \& Technology Co., Ltd.) $48 \mathrm{~h}$ subsequent to infection.

Colony formation assay. SK-MEL-1 and WM-115 cells infected with miR-33a or negative control lentivirus were plated on a 6-well plate (500 cells/well). Medium was refreshed every 2 days. The cells were fixed and stained with $0.1 \%$ crystal violet in Eagle's minimum essential medium (Solarbio Science $\&$ Technology Co., Ltd.), and 2 weeks later, the colonies containing $>50$ cells were counted as a colony. Colonies were counted in 5 fields of view at a magnification of $x 40$ using a light microscope (BX51; Olympus Corporation, Tokyo, Japan). Experiments were performed $\geq 3$ times independently.

Cell proliferation. Cell proliferation was examined using a bromodeoxyuridine (BrdU) incorporation assay and anaphase analysis. The BrdU incorporation rate was measured by a BrdU Cell Proliferation ELISA kit (Abcam, Cambridge, UK), following the manufacturer's protocol. For anaphase analysis, the number of cells and the number of cells in anaphase were detected by 4',6-diamidino-2-phenylindole staining (Solarbio Science \& Technology Co., Ltd.) and were counted in 3 fields of view per well at a magnification of $x 20$ using an Olympus IX83 microscope (Olympus Corporation).

Luciferase reporter assay. For the luciferase activity assay, a wild-type 3'-UTR segment of PCTAIRE1 was cloned into a pmirGLO plasmid (Promega Corporation, Madison, WI, USA). The mutated sequence in the complementary site for miRNA-33a was generated by site-specific mutagenesis. All transfections were performed using Invitrogen Lipofectamine ${ }^{\circledR} 2000$ (Thermo Fisher Scientific, Inc.), according to the manufacturer's protocol. The cells were plated on a 24-well plate (Corning Incorporated) and co-transfected with either a wild-type or mutant 3'-UTR segment of PCTAIRE1 and a Renilla luciferase reporter (Promega Corporation). In total, $48 \mathrm{~h}$ subsequent to transfection, luciferase activity was measured by a dual-luciferase reporter assay system (Promega Corporation). Renilla luciferase activity was used as an internal reference. Experiments were performed $\geq 3$ times independently.

Extraction of $m R N A$ and miRNA and reverse transcription-quantitative polymerase chain reaction (RT-qPCR). Total RNA samples were isolated using the RNA Mini kit (Qiagen China Co., Ltd., Shanghai, China). An Applied Biosystems Taqman miRNA assay kit and Taqman miRNA assay (Thermo Fisher Scientific, Inc.) were used to quantify the expression of mature miRNAs, according to the manufacturer's protocol. Fold changes were calculated using the $\Delta \Delta \mathrm{Cq}$ method (23). The Taqman miRNA assay was used to quantify mature miRNA expression. U6 miRNA was used as an internal reference for miRNA expression. mRNA expression was measured by RT-qPCR using the Applied Biosystems Taq Man Universal PCR Master Mix (Thermo Fisher Scientific, Inc.). Samples were analyzed using Applied Biosystems StepOnePlus $^{\mathrm{TM}}$ Real-Time PCR System (Thermo Fisher Scientific, Inc.).

Protein extraction and western blot assay. Whole-cell protein lysates were prepared by removing the medium, washing the cells with phosphate-buffered saline (Gibco; Thermo Fisher Scientific, Inc.), scraping the cells from the plates and pelleting the cells by centrifugation at $700 \mathrm{x} g$ for $10 \mathrm{~min}$ (Centrifuge 5418R; Eppendorf North America, Hauppauge, NY, USA). The cell pellets were resuspended in radioimmunoprecipitation assay buffer (Solarbio Science \& Technology Co., Ltd.), which contained a protease inhibitor cocktail and a phosphatase inhibitor cocktail (Roche Diagnostics, Basel, Switzerland). Subsequent to protein lysis, western blot analysis was performed. Primary monoclonal rabbit anti-human PCTAIRE1 antibody (dilution, 1:1,000; catalog no., 4852) and rabbit anti-human p27 antibody (dilution, 1:1,000; catalog no., 3686) were purchased from CST Biological Reagents Co., Ltd. (Shanghai, China). Polyclonal rabbit anti-human p27 KIP1 antibody (phospho T187; dilution, 1:1,000; catalog no., ab75908) was obtained from Abcam and polyclonal rabbit anti-human glyceraldehyde 3-phosphate dehydrogenase antibody (dilution, 1:10,000; catalog no., G9545) was from Sigma-Aldrich (St. Louis, MO, USA).

Statistical analysis. The significance of differences was analyzed using two-tailed Student's $t$-test using Prism 6 software (GraphPad Software, Inc., La Jolla, CA, USA). All data are presented as the mean \pm standard deviation from $\geq 3$ separate experiments. $\mathrm{P}<0.05$ was considered to indicate a statistically significant difference.

\section{Results}

miR-33a is downregulated in melanoma cells and negatively regulates cell proliferation. To explore the functional role of miR-33a, the present study first examined the expression of miR-33a in melanocyte and melanoma cell lines. The RT-qPCR assay results revealed that miR-33a exhibited decreased expression in melanoma cell lines, particularly in SK-MEL-1 and WM-115 cells, compared with melanocyte cells (Fig. 1A). These results indicate that miR-33a may be lost in melanoma development. The present study infected SK-MEL-1 and WM-115 cells with miR-33a-expressing lentivirus. miR-33a overexpression was confirmed by an RT-qPCR assay (Fig. 1B) and a colony formation assay was performed. As revealed in Fig. 1C and D, the infection of miR-33a-expressing lentivirus significantly suppressed colony numbers and the size of SK-MEL-1 and WM-115 cells. These results suggest that miR-33a may affect tumorigenesis of melanoma cells. Furthermore, the present study aimed to investigate the effect of miR-33a overexpression on the proliferation of melanoma cells. To investigate the effect of miR-33a on cell proliferation, a BrdU incorporation assay was performed. In SK-MEL-1 and WM-115 cells, miR33a overexpression significantly reduced the BrdU incorporation 
A

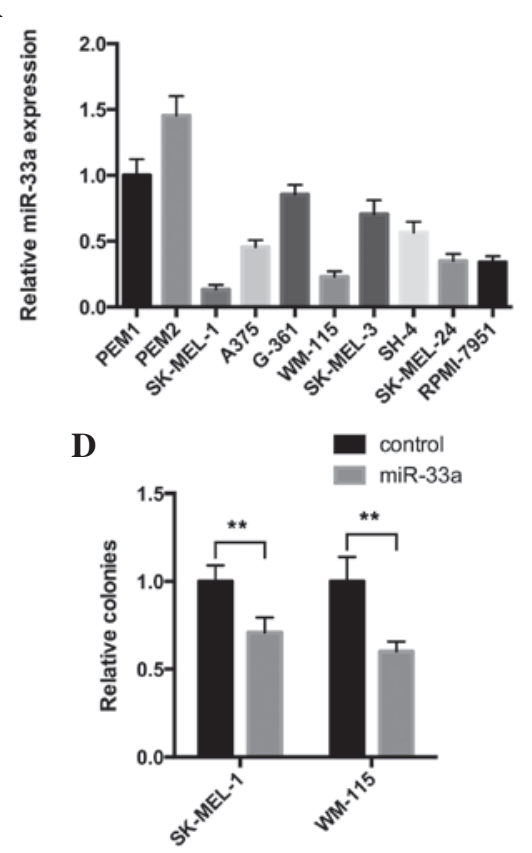

B
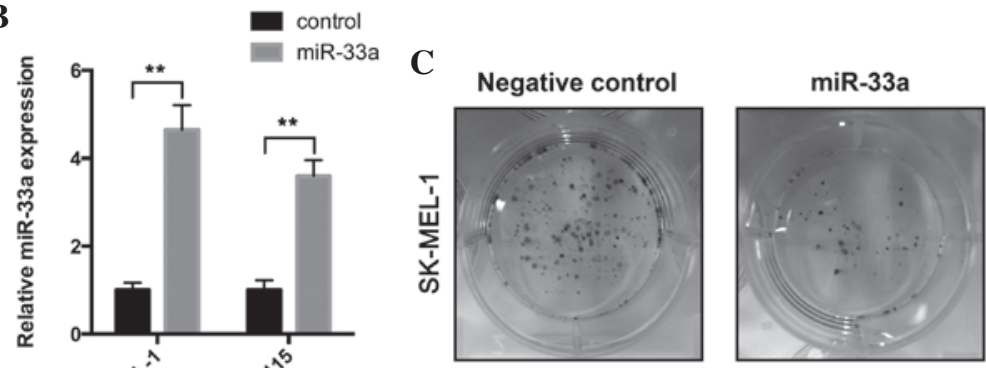

$\mathbf{E}$
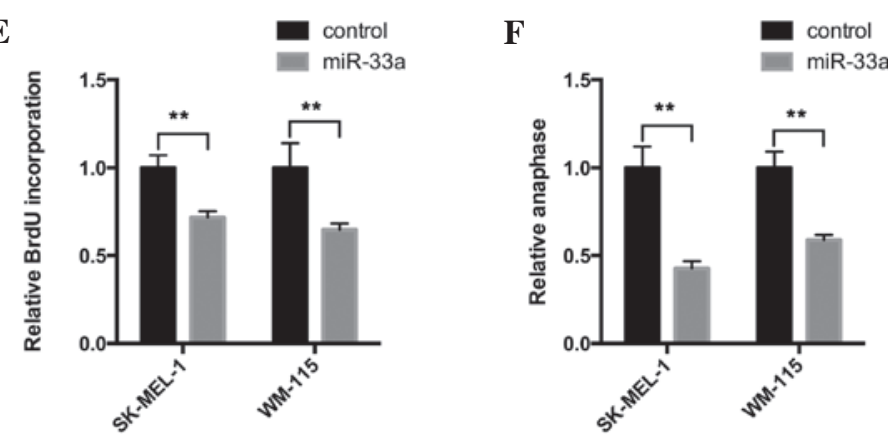

Figure 1. miR-33a is downregulated in melanoma cell lines and suppresses cell proliferation. (A) The expression pattern of miR-33a in various melanocyte and melanoma cell lines was examined by RT-qPCR. (B) Human melonoma SK-MEL-1 and WM-115 cells were infected with miR-33a-expressing or negative control lentivirus. The overexpression efficiency of miR-33a was examined by RT-qPCR. (C and D) Stable melanoma cells overexpressing miR-33a and negative control cells were subjected to colony formation assay. (C) Representative colonies of SK-MEL-1 cells. (D) Relative colony numbers exhibted by histogram. To investigate the impact of miR-33a on cell proliferation, melanoma cells infected with miR-33a expressing and negative control lentivirus were subjected to (E) BrdU incorporation assay and (F) anaphase analysis. Error bars indicate the standard deviation. Experiments were performed three times. ${ }^{* *} \mathrm{P}<0.01$. miR-33a, microRNA-33a; RT-qPCR, reverse transcription-quantitative polymerase chain reaction; BrdU, bromodeoxyuridine.

rate (Fig. 1E), which demonstrated that cell proliferation was suppressed. Anaphase analysis was also performed to confirm the suppressive role of miR-33a on proliferation. Similarly, miR-33a-overexpressing cells exhibited decreased anaphase cell numbers compared with the negative control cells (Fig. 1F). Overall, the present data indicate that miR-33a has a tumor suppressive role in melanoma cells.

miR-33a targets the 3'-UTR of PCTAIRE1 mRNA and downregulates PCTAIRE1 expression in melanoma cells. miRNAs exert their biological functions by marking the target transcript for degradation (24). To determine potential miR-33a targets involved in melanoma proliferation, the present study used a combination of bioinformatic tools for miRNA target prediction. TargetScan (Whitehead Institute for Biomedical Research, Cambridge, MA, USA; available from http://www.targetscan.org/), PicTar (Center for Comparative Functional Genomics, New York, NY, USA and the Max Delbruck Centrum, Berlin, Germany; available from http://pictar.mdc-berlin.de/) and miRanda (Memorial Sloan-Kettering Cancer Center, New York, NY, USA; available from http://www.microrna.org/microrna/home.do) algorithms were used to predict miR-33a targets. PCTAIRE1 was revealed as a potential miR-33a target (Fig. 2A), and has been reported as a cell cycle regulator in hepatocellular carcinoma and breast cancer (12). Therefore, the present study hypothesized that miR-33a may inhibit melanoma proliferation by directly targeting PCTAIRE1. To determine whether miR-33a targets this predicted target gene, the present study generated reporter constructs with the luciferase coding sequence fused to the wild-type or mutant miR-33a binding sequence of
PCTAIRE1 3'-UTRs (Fig. 2A). Melanoma cells infected with miR-33a-expressing lentivirus or negative control lentivirus were transfected with reporter constructs. The present results demonstrated that miR-33a overexpression significantly repressed the activity of the wild-type reporter construct in SK-MEL-1 and WM-115 cells (Fig. 2B). Furthermore, miR-33a had no repressive effect on reporter vectors carrying the mutated PCTAIRE1 3'-UTR (Fig. 2B). These results indicate that miR-33a may directly interact with the PCTAIRE1 3'-UTR. To confirm the negative effect that miR-33a exerts on PCTAIRE1 expression, the present study examined PCTAIRE1 expression in cells overexpressing miR-33a and negative control cells. As revealed by Fig. $2 \mathrm{C}$ and $\mathrm{D}$, the mRNA and protein forms of PCTAIRE1 were downregulated following miR-33a overexpression. Together, the present data suggest that PCTAIRE1 is a target of miR-33a.

miR-33a suppresses p27 phosphorylation and upregulates p27 expression in melanoma cells by targeting PCTAIRE1. p27 is a tumor suppressor that regulates the proliferation, motility and apoptosis of cells (25). A recent study demonstrated that PCTAIRE1 phosphorylates p27 and enhances the degradation of p27, therefore promoting cell proliferation (22). The present study hypothesized that miR-33a may exert its tumor suppressive function by preventing PCTAIRE1-induced phosphorylation of p27. To test this hypothesis, the present study first examined whether $\mathrm{p} 27$ expression was controlled by miR-33a. As expected, RT-qPCR revealed that $\mathrm{p} 27$ transcripts were highly upregulated in melanoma cells infected with miR-33a-expressing lentivirus compared with cells infected with negative control lentivirus (Fig. 3A). In accordance with these results, a western blot assay 
A

\section{PCTAIRE1}

WT 3'UTR 5'...UUUUUGUUUUUUUUAAAUGCAAU....3' miR-33a ACGUUACGUUGAUGUUUACGUG MUT 3'UTR 5'...UUUUUGUUUUUUUUAACGUGAAU...3'

C

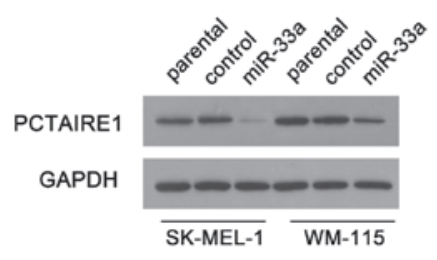

\section{B ஃ}

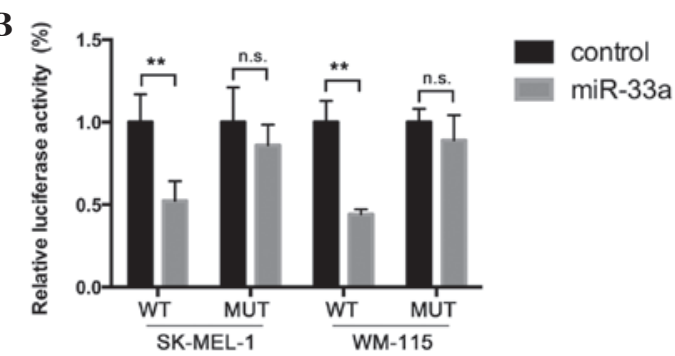

D

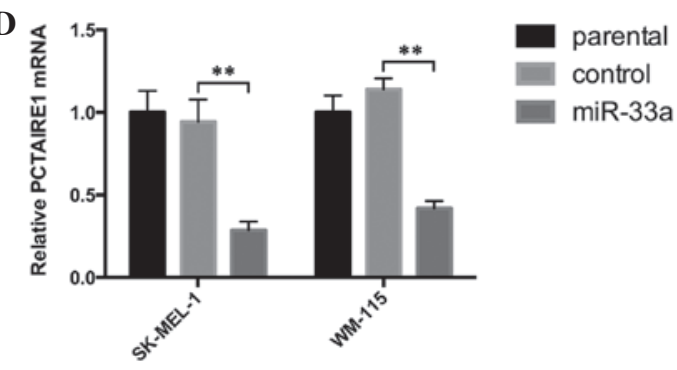

Figure 2. PCTAIRE1 is a direct target of miR-33a in melanoma cells. (A) Putative miR-33a complementary site in the 3'-UTR of PCTAIRE1 mRNA. A mutated 3'-UTR of PCTAIRE1 mRNA for the miR-33a complementary site was generated. (B) Melanoma cells infected with miR-33a expressing and negative control lentivirus were co-transfected with luciferase reporter plasmid with either WT or MUT and Renilla luciferase reporter. In total, $48 \mathrm{~h} \mathrm{later,} \mathrm{lucif-}$ erase activity was measured and Renilla luciferase activity was used as an internal reference. (C and D) The effect of miR-33a on PCTAIRE1 expression was measured by (C) western blot assay and (D) reverse transcription-quantitative polymerase chain reaction. Error bars indicate the standard deviation. Experiments were performed three times. ${ }^{* *} \mathrm{P}<0.01$. miR-33a, microRNA-33a; mRNA, messenger RNA; UTR, untranslated region; WT, wild-type; MUT, mutant PCTAIRE1 3'-UTR.

A

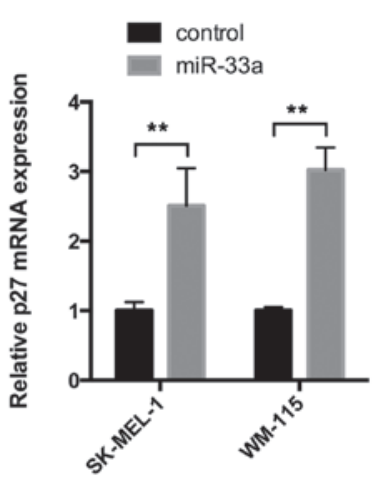

B

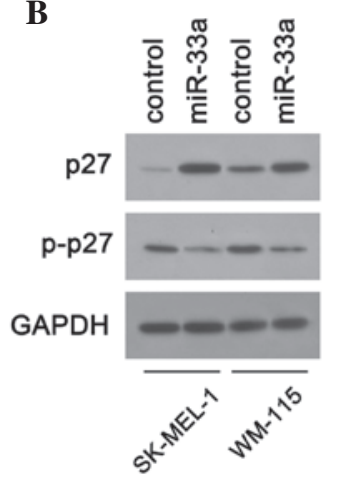

C
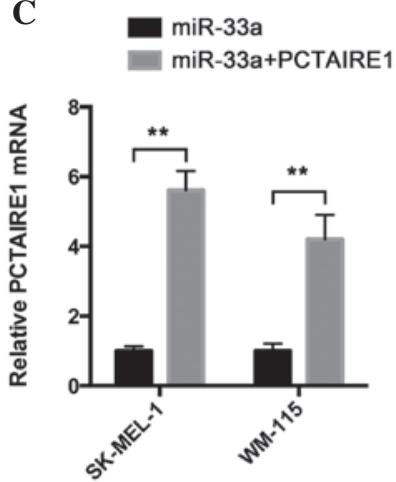

D

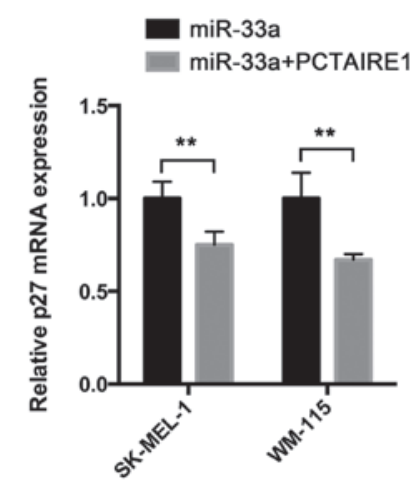

$\mathbf{E}$
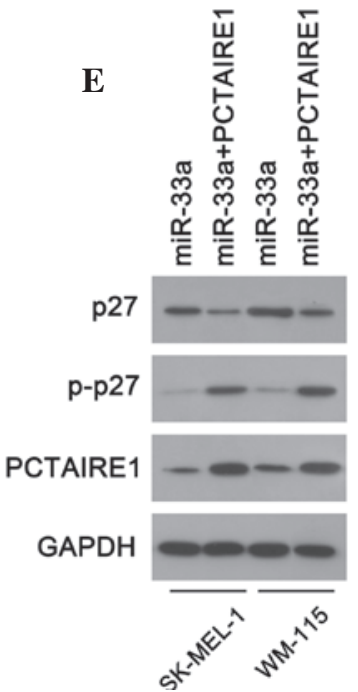

Figure 3. miR-33a suppresses p27 phosphorylation and upregulates its expression in melanoma cells by targeting PCTAIRE1. (A and B) p27 expression and p27 phosphorylation in cells overexpressing miR-33a or negative control cells were measured by (A) RT-qPCR and (B) western blot assay. (C and D) Cells overexpressing miR-33a were transfected with PCTAIRE1 or a vector construct. In total, $48 \mathrm{~h}$ later, RT-qPCR was used to examine (C) PCTAIRE1 mRNA expression and (D) p27 expression and phosphorylation alteration. (E) Western blot assay of p27 expression and phosphorylation. Error bars indicate the standard deviation. Experiments were performed three times. ${ }^{* *} \mathrm{P}<0.01$. miR-33a, microRNA-33a; RT-qPCR, reverse transcription-quantitative polymerase chain reaction; mRNA, messenger RNA; GAPDH, glyceraldehyde 3-phosphate dehydrogenase. 


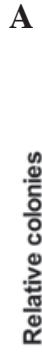

miR-33a
miR-33a+PCTAIRE1

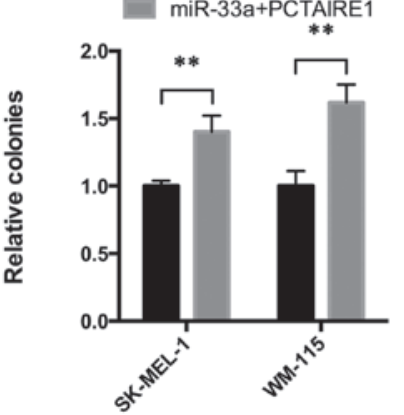

B
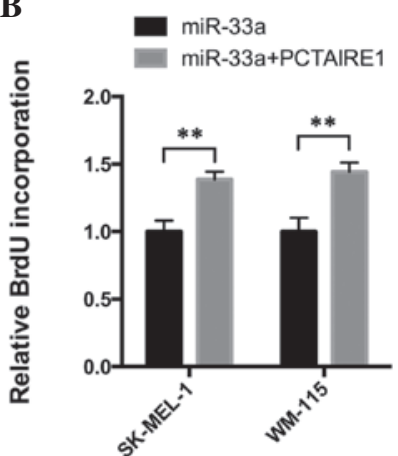

C

miR-33a

miR-33a+PCTAIRE1

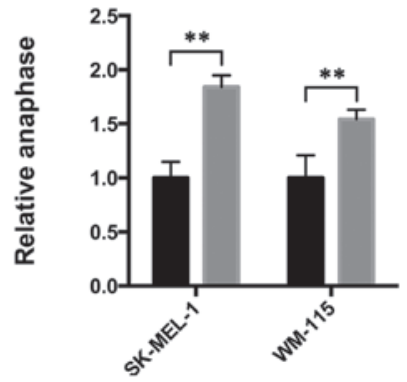

Figure 4. PCTAIRE1 overexpression reversed miR-33a-induced colony formation and cell proliferation inhibition. Cells overexpressing miR-33a were transfected with PCTAIRE1 or a vector construct. The cells were subjected to (A) colony formation assay, (B) BrdU incorporation assay or (C) anaphase analysis. Error bars indicate the standard deviation. Experiments were performed three times. ${ }^{* *} \mathrm{P}<0.01$. miR-33a, microRNA-33a; BrdU, bromodeoxyuridine.

confirmed the upregulated protein expression of PCTAIRE1 following miR-33a overexpression (Fig. 3B). Phosphorylation of p27 leads to its degradation and promotes the activation of assembled cyclin D-cyclin dependent kinases (25). Therefore, the present study examined the phosphorylation state of p27. The present results demonstrated that cells overexpressing miR-33a exhibited less phosphorylated p27 compared with negative control cells (Fig. 3B). Furthermore, the present study examined whether miR-33a modulates p27 phosphorylation and accumulation by targeting the PCTAIRE1 gene. The present study overexpressed PCTAIRE1 in cells overexpressing miR-33a by transfecting the miR-33a cells with a PCTAIRE1 expression plasmid. RT-qPCR and a western blot assay were performed to confirm PCTAIRE1 overexpression (Fig. 3C and E). As expected, enforced PCTAIRE1 expression reversed miR-33a induced p27 accumulation (Fig. 3D and E). p27 phosphorylation was also upregulated (Fig. 3E). Overall, the present data indicate that miR-33a may regulate $\mathrm{p} 27$ by targeting PCTAIRE1, and therefore may inhibit cell proliferation.

PCTAIREl overexpression reverses miR-33a-induced colony formation and cell proliferation inhibition. The aforementioned results indicate that miR-33a may demonstrate tumor suppressor activity by downregulating PCTAIRE1 and upregulating p27. To investigate this hypothesis, the present study overexpressed PCTAIRE1 in cells overexpressing miR-33a (Fig. 3C and E) and a colony formation assay was performed. As expected, in SK-MEL-1 and WM-115 cells, PCTAIRE1 overexpression significantly reversed the miR-33a-induced tumor suppressive effects (Fig. 4A). Furthermore, the BrdU incorporation rate was increased following PCTAIRE1 overexpression (Fig. 4B). In addition, the number of cells in anaphase was also increased in
PCTAIRE1-overexpressing cells (Fig. 4). Overall, the present results confirmed that miR-33a demonstrates tumor suppressive activity by targeting PCTAIRE1.

\section{Discussion}

miR-33a has been previously identified as a lipid regulator that controls the cellular balance of cholesterol and fatty acid metabolism (8-11). The present study demonstrated that miR-33a is downregulated in melanoma cells compared with melanocytes. Knockdown of miR-33a enhanced colony formation and cell proliferation in melanoma cells. Therefore, the present study reveals that miR-33a acts as a novel tumor suppressor in melanoma.

PCTAIRE1 is widely expressed in human tissue and certain studies suggest that PCTAIRE1 participates in various biological processes, including insulin secretion, secretory cargo transport and dendrite development $(19,20,26)$. The role that PCTAIRE1 plays in tumor progression has been recently revealed; PCTAIRE1 phosphorylates p 27 and regulates mitosis in cancer cells (22). However, the mechanism by which PCTAIRE1 is regulated is unclear. Although certain miR-33a targets have been identified, its direct target in melanoma and the mechanism by which miR-33a exerts its tumor suppressive behavior remains unknown. The present study demonstrated that PCTAIRE1 is negatively regulated by the novel miRNA miR-33a. By performing a luciferase reporter assay and western blot assays, the present study demonstrated that miR-33a directly targets PCTAIRE1 in human melanoma SK-MEL-1 and WM-115 cells. These results provide novel information concerning the mechanisms by which miR-33a and PCTAIRE1 act. 
p27, also termed KIP1 or p27 $7^{\text {kip }}$, is a one of the most widely studied tumor suppressors (25). It has been reported that p27 regulates cell proliferation, motility and apoptosis (25,27-29). The present study revealed that $\mathrm{p} 27$ is upregulated by miR-33a, and this may be due to miR-33a reducing the level of phosphorylated p27. Furthermore, the present study confirmed that PCTAIRE1 negatively regulates p27 expression in melanoma cells and the effects of miR-33a on p27 may be dependent on miR-33a directly targeting PCTAIRE1.

In summary, the present analysis reveals that miR-33a is an important negative regulator of cell proliferation. In addition, the present study demonstrated that PCTAIRE1 is a target of miR-33a in melanoma cells. Furthermore, miR-33a maintains p27 expression by targeting PCTAIRE1. Overexpression of PCTAIRE1 reversed the miR-33a-induced suppression of cell proliferation. Overall, the present findings indicate that miR-33a acts as a tumor suppressor in melanoma cells.

\section{References}

1. Siegel R, Ma J, Zou Z and Jemal A: Cancer statistics, 2014. CA Cancer J Clin 64: 9-29, 2014

2. Miller AJ and Mihm MC Jr: Melanoma. N Engl J Med 355: 51-65, 2006.

3. Gray-Schopfer V, Wellbrock C and Marais R: Melanoma biology and new targeted therapy. Nature 445: 851-857, 2007.

4. Calin GA and Croce CM: MicroRNA signatures in human cancers. Nat Rev Cancer 6: 857-866, 2006.

5. John B, Enright AJ, Aravin A, Tuschl T, Sander C and Marks DS: Human MicroRNA targets. PLoS Biol 2: e363, 2004.

6. Nishikawa S, Ishii H, Haraguchi N, Kano Y, Fukusumi T, Ohta K, Ozaki M, Dewi DL, Sakai D, Satoh T, et al: microRNA-based cancer cell reprogramming technology. Exp Ther Med 4: 8-14, 2012.

7. Ha M and Kim VN: Regulation of microRNA biogenesis. Nat Rev Mol Cell Biol 15: 509-524, 2014

8. Gerin I, Clerbaux LA, Haumont O, Lanthier N, Das AK, Burant CF, Leclercq IA, MacDougald OA and Bommer GT: Expression of miR-33 from an SREBP2 intron inhibits cholesterol export and fatty acid oxidation. J Biol Chem 285: 33652-33661, 2010.

9. Marquart TJ, Allen RM, Ory DS and Baldán A: miR-33 links SREBP-2 induction to repression of sterol transporters. Proc Nat Acad Sci USA 107: 12228-12232, 2010.

10. Najafi-Shoushtari SH, Kristo F, Li Y, Shioda T, Cohen DE, Gerszten RE and Näär AM: MicroRNA-33 and the SREBP host genes cooperate to control cholesterol homeostasis. Science 328: $1566-1569,2010$

11. Dávalos A, Goedeke L, Smibert P, Ramírez CM, Warrier NP, Andreo U, Cirera-Salinas D, Rayner K, Suresh U, Pastor-Pareja JC, et al: $\mathrm{miR}-33 \mathrm{a} / \mathrm{b}$ contribute to the regulation of fatty acid metabolism and insulin signaling. Proc Natl Acad Sci USA 108: 9232-9237, 2011.

12. Cirera-Salinas D, Pauta M, Allen RM, Salerno AG, Ramírez CM, Chamorro-Jorganes A, Wanschel AC,Lasuncion MA, Morales-Ruiz M, Suarez Y, et al: Mir-33 regulates cell proliferation and cell cycle progression. Cell Cycle 11: 922-933, 2012.
13. Takwi AA, Li Y, Becker Buscaglia LE, Zhang J, Choudhury S, Park AK, Liu M, Young KH, Park WY, Martin RC and Li Y: A statin-regulated microRNA represses human c-Myc expression and function. EMBO Mol Med 4: 896-909, 2012.

14. Thomas M, Lange-Grünweller K, Weirauch U, Gutsch D, Aigner A, Grünweller A and Hartmann RK: The proto-oncogene Pim-1 is a target of miR-33a. Oncogene 31: 918-928, 2012

15. Rice SJ, Lai SC, Wood LW, Helsley KR, Runkle EA, Winslow MM and Mu D: MicroRNA-33a mediates the regulation of high mobility group AT-hook 2 gene (HMGA2) by thyroid transcription factor 1 (TTF-1/NKX2-1). J Biol Chem 288: 16348-16360, 2013.

16. Meyerson M, Enders GH, Wu CL, Su LK, Gorka C, Nelson C, Harlow E and Tsai LH: A family of human cdc2-related protein kinases. EMBO J 11: 2909-2917, 1992.

17. Okuda T, Cleveland JL and Downing JR: PCTAIRE-1 and PCTAIRE-3, two members of a novel cdc2/CDC28-related protein kinase gene family. Oncogene 7: 2249-2258, 1992.

18. Yeung ML, Houzet L, Yedavalli VS and Jeang KT: A genome-wide short hairpin RNA screening of jurkat T-cells for human proteins contributing to productive HIV-1 replication. J Biol Chem 284: 19463-19473, 2009

19. Fu WY, Cheng K, Fu AK and Ip NY: Cyclin-dependent kinase 5-dependent phosphorylation of Pctairel regulates dendrite development. Neuroscience 180: 353-359, 2011.

20. Chen XY, Gu XT, Saiyin H, Wan B, Zhang YJ, Li J, Wang YL, Gao R, Wang YF, Dong WP, et al: Brain-selective kinase 2 (BRSK2) phosphorylation on PCTAIRE1 negatively regulates glucose-stimulated insulin secretion in pancreatic $\beta$-cells. J Biol Chem 287: 30368-30375, 2012.

21. Hill SJ, Rolland T, Adelmant G, Xia X, Owen MS, Dricot A, Zack TI, Sahni N, Jacob Y, Hao T, et al: Systematic screening reveals a role for BRCA1 in the response to transcription-associated DNA damage. Genes Dev 28: 1957-1975, 2014.

22. Yanagi T, Krajewska M, Matsuzawa S and Reed JC: PCTAIRE1 phosphorylates p27 and regulates mitosis in cancer cells. Cancer Res 74: 5795-5807, 2014.

23. Livak KJ and Schmittgen TD: Analysis of relative gene expression data using real-time quantitative PCR and the 2(-Delta Delta C(T)) Method. Methods 25: 402-408, 2001.

24. Cai Y, Yu X, Hu S and Yu J: A brief review on the mechanisms of miRNA regulation. Genomics Proteomics Bioinformatics 7: 147-154, 2009.

25. Chu IM, Hengst L and Slingerland JM: The Cdk inhibitor p27 in human cancer: Prognostic potential and relevance to anticancer therapy. Nat Rev Cancer 8: 253-267, 2008.

26. Palmer KJ, Konkel JE and Stephens DJ: PCTAIRE protein kinases interact directly with the COPII complex and modulate secretory cargo transport. J Cell Sci 118: 3839-3847, 2005.

27. Vervoorts J and Lüscher B: Post-translational regulation of the tumor suppressor p27(KIP1). Cell Mol Life Sci 65: 3255-3264, 2008.

28. Lee SR, Shin JW, Kim HO, Son BH, Yoo CH and Shin JH: Determining the effect of transforming growth factor- $\beta 1$ on cdk4 and p27 in gastric cancer and cholangiocarcinoma. Oncol Lett 5: 694-698, 2013.

29. Zhang M, Li J, Wang L, Tian Z, Zhang P, Xu Q, Zhang C, Wei F and Chen W: Prognostic significance of p21, p27 and survivin protein expression in patients with oral squamous cell carcinoma. Oncol Lett 6: 381-386, 2013. 